\title{
A Cyberphysical Learning Approach for Digital Smart Citizenship Competence Development
}

\author{
Yacine Atif \\ University of Skövde \\ Skövde, Sweden \\ yacine.atif@his.se \\ Demetrios Sampson \\ Curtin University \\ Perth, Australia \\ demetrios.sampson@curtin.edu.au
}

\author{
Stylianos Sergis \\ University of Piraeus \\ Athens, Greece \\ steliossergis@gmail.com \\ Gunnar Mathiason \\ University of Skövde \\ Skövde, Sweden \\ gunnar.mathiason@his.se
}

\begin{abstract}
Smart Cities have emerged as a global concept that argues for the effective exploitation of digital technologies to drive sustainable innovation and well-being for citizens. Despite the large investments being placed on Smart City infrastructure, however, there is still very scarce attention on the new learning approaches that will be needed for cultivating Digital Smart Citizenship competences, namely the competences which will be needed by the citizens and workforce of such cities for exploiting the digital technologies in creative and innovative ways for driving financial and societal sustainability. In this context, this paper introduces cyberphysical learning as an overarching model of cultivating Digital Smart Citizenship competences by exploiting the potential of Internet of Things technologies and social media, in order to create authentic blended and augmented learning experiences.
\end{abstract}

\section{Keywords}

Digital Smart citizenship; smart city; smart citizenship competences, cyberphysical systems; learning design; social networks; learning technology; smart grid; collaborative learning; Internet of Things.

\section{INTRODUCTION}

Smart Cities refer to cities that use digital technologies effectively to enhance their overall functioning and to engage more effectively and actively with their citizens $[7,8]$. Smart Cities have emerged as a global need and challenge due to the understanding that more than $54 \%$ of the global population now resides within cities, with an estimation that this ratio will increase to $66 \%$ by 2050 [19]. This situation places city administrators against significant and com-

(C)2017 International World Wide Web Conference Committee (IW3C2), published under Creative Commons CC BY 4.0 License.

WWW 2017 Companion, April 3-7, 2017, Perth, Australia. ACM 978-1-4503-4914-7/17/04.

http://dx.doi.org/10.1145/3041021.3054167

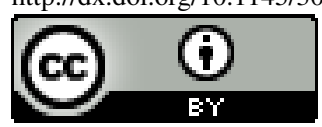

plex challenges related with ensuring not only the well-being of the citizens and the environment protection, but also financial and societal sustainability through innovation. In this context, the concept of Smart Cities has emerged as a potential solution, receiving a significant level of attention globally and forging an emerging research and innovation field [5] using their own surrounding environment as a living laboratory. In this context, education for Smart Cities needs to be focusing on such cultivating Digital Smart Citizenship competences, including digital literacy, communication and collaboration and creative problem solving, towards increased productivity and the capacity for self-driven behavioral changes. Subsequently, new levels of instruction beyond traditional classroom contexts need to be defined to support cultivation of such competences, and commodity offerings are packaged with consumer's education to adopt some behavioral changes using emerging digital technologies.

In Smart Cities, the use of emerging technologies is used to drive sustainability and innovation in diverse aspects of the city functioning, such as resource management, transportation and governance [9]. This approach also requires citizens to adopt new lifestyles and attain new competences that prompt superior sustainability values and allow them to engage in sustainable innovation capacity to solve illdefined problems by exploiting digital technologies. Such Digital Smart citizenship competences provide citizens with the capacity to not only effectively participate in their Smart City's development but also influence it and change the world around them

The use of emerging technologies are driving companies to shift features of value-added business services from "nice to have" to "must have". This imperative brings seed citizens to adopt new lifestyles that prompt superior sustainability values. The process is facilitated by providing citizens with opportunities through which they learn how to change the world around them [5] using their own surrounding environment as a living laboratory.

Internet of Things (IoT) is one of these technologies that are expected to lead to behavioural changes across a range of applications of Smart City function. To conceptualize the preparation for a smarter level of digital smart citizenship, IoT unleashes the instructional power hidden within physical resources and provide learning interfaces through translating real-time data readings into contextual diagnosis. Data 


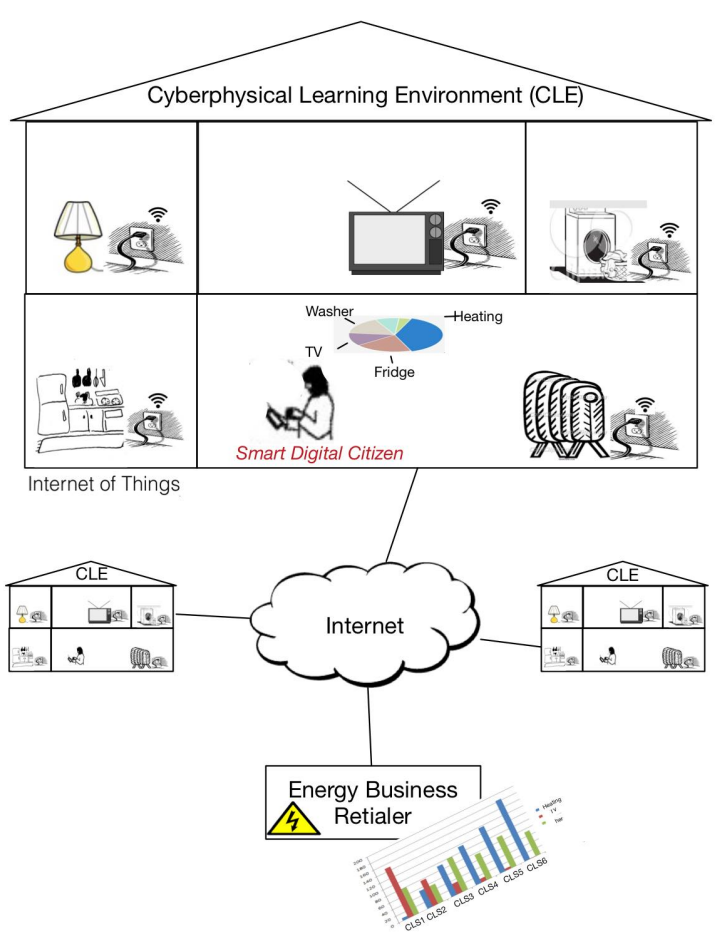

Figure 1. User engagement in smart grid

streamed from these resources is visualized to learn about sustainable assets hidden within physical phenomena, via personalized and context-driven instructional paths. This approach leverages a new dimension to situated learning and aggregates the fluid connectedness between citizens and physical resources across programmable environments embedded with information technologies.

For example, trends such as connected cars, connected appliances, smart grid etc. require citizens engagement to rethink sustainable transitions within the so called "circulareconomy" [18] market. Thus, citizens as consumers play a pivotal role towards these transitions through everyday objects that signal their identity and leverage their digital smart citizenship, but require a learning curve to engage with presumably passive and lifeless objects. Connected objects are creating unprecedented sources of information, which could be analyzed to recognize personalized patterns and incite behavioural changes, taking humanity to smarter digital era shaped by the prospective circular economy. A network of devices connects physical objects to deploy services that immerse learners into a social cyberphysical ecosystem. In this ecosystem, tangible education is delivered to influence progressive behavioral changes that are mutually beneficial to learners and service providers.

As an illustration, consider household energy data which can be harnessed from smart meters and smart plugs, providing a rich data source of appliances usage. These data sources educate consumers on home's economics and provide energy retailers with a range of marketing implications: which appliance has been used, for how long, and in what mode for which period of the week/month/year? All this data is seamlessly collected to engage energy-aware consumers, allowing retailers advocating new marketing strategies, induced by incentives in order to balance energy-load, as illustrated in Figure 1. Retailers would rather redistribute energy-excess (across time) than buying shortages from energy suppliers (at a cost) or building new power distribution infrastructure, in a scheme known as Demand-Response used to schedule load in a way that accommodates the available capacity. However, this endeavor requires consumers' awareness and engagement which are achieved through a new cycle of education processes. Uneducated consumers reduce their smart digital citizenship footprint and delay sustainability shifts as, for example, evidenced by a pilot study in the UK done throughout 2014-2015 [9], which found three groups of households with similar characteristics suggesting similar energy profile. Yet, one of the groups had three times the energy consumption. Further analysis revealed higher energy consumption for washing machine usage, when using cotton cycles $100 \%$ of the time against $40-50 \%$ of the time in the other groups. IoT induced energy data could have identified these patterns to devise appropriate educative schemes, in this case, use of economy cycles, promoted by manufacturers or (retail energy) service providers. The problem is that business entities are mostly wary about, and expert in the product or the service they deliver, and lack the education focus in terms of pedagogical models and learning processes. This example further highlights the challenge that apart from the investments on designing and implementing Smart Cities, significant effort and attention needs to be placed on cultivating the required Digital Smart Citizenship competences that will allow citizens and workforce to exploit digital technologies within their city and actively contribute to its sustainable development. The combination of IoT, social media and mobile data are paving the way to such new personalized education dimensions, which in turn could be turned into new personalized marketing opportunities.

In this context, this paper proposes a cyber-physical continuum for supporting the cultivation of digital smart citizenship competences, where a spectrum of blended and augmented experiences offer new opportunities for nurturing progressive behavioral change cycles. Our approach collects information seamlessly from citizens' behaviors (in the case study of this work such behaviors will be consumers' use of nondigital appliances) and enables a transformation of learning capacity from clumsy quantitative to judicious qualitative reasoning, that progressively qualify learners to a higher degree in sustainability dispositions, and digital smart citizenship.

\section{SMART CITIES}

Even though a unifying definition of Smart Cities has not yet been proposed [6], they can be broadly defined as those cities that make "use of information and communication technology to sense, analyze and integrate the key information of core systems in running cities" (IBM as cited in [6]). More specifically, Smart Cities exploit a wide range of digital technologies [15] to improve their functioning across a set of commonly acknowledged dimensions [1]:

- Smart Economy, namely financial competitiveness of a city through innovation, productivity and flexibility of the workforce and the market. 
- Smart People, namely consideration for improving the learning, creative and collaboration capacity of human capital.

- Smart Living, namely enhancing social and cultural cohesion and the quality of services to citizens such as housing, health and education.

- Smart Mobility, namely promoting accessible and innovative transportation and communication infrastructure.

- Smart Governance, namely promoting inclusion and participation of citizens in the city's decision making.

- Smart Environment, namely promoting effective and sustainable use of natural resources.

As aforementioned, Smart Cities are currently receiving a significant level of attention globally and vast investments are being allocated on researching and implementing Smart City initiatives [14]. However, as aforementioned, apart from the large public and private investments on Smart Cities infrastructure, in order to holistically introduce Smart Cities in the fabric of society, it is also important to invest in appropriately preparing the human capital of such cities.

More specifically, focus should be placed on preparing the future citizens and workforce of such cities in terms of core competences which will be needed for driving innovation and sustainability across the different dimensions of the city functions. The required Digital Smart Citizenship competences, therefore, will need to comprise diverse skillsets including digital literacy, communication and collaboration, creative problem solving as well as inquiry skills for collecting, processing and evaluating information and data (e.g., $[14,12])$.

The main standpoint of this paper is that conventional learning approaches may be inefficient to prepare and sustain such Digital Smart Citizenship competences if they do not explicitly build on situated learning paradigms that exploit emerging smart technologies (e.g., IoT technologies) that are essentially the backbone of Smart Cities. Therefore, to address this issue, this paper describes the concept of cyberphysical learning, as a strand of situated learning that explicitly comprises social aspects supported by smart IoT technologies.

\section{THE CONCEPT OF CYBERPHYSICAL LEARNING}

Cyberphysical learning comprises instructional dimensions that can be used to build digital smart citizenship competences by rendering situational learning as a function of an activity and context, across an instructional scaffolding approach. This form of learning contrasts with traditional classroom instruction where knowledge is abstract and out of context. Social interaction is a critical component of situated learning whereby learners become involved in a "community of practice" that embodies targeted beliefs and behaviors to be acquired. As beginners or newcomers move from the periphery of this community to its center, they become more active and engaged within the culture of the community and progressively adopt the desired behavior.

Cyberphysical learning shares the same properties as situated learning but community participants may include physical resources used as cognitive tools and blended augmented experiences in an authentic domain activity [3]. Namely, given learner state $S$, we say that cyberphysical learning occurs when a physical stimulus event $E_{i}$ maximizes the probability that a learner moves its behavioral state to S'. Hence, cyberphysical learning is distinguished by physical events associated with behavioral-impacts likelihood. Formally, this likelihood is modeled by the following probability equation:

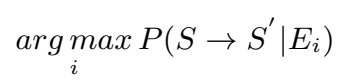

In addition, the probability that the learner adopts the behavioral state $S^{\prime}$ under stimulus $E_{i}$ is strictly greater than the probability that $S$ changes its state independently, namely:

$$
P\left(S \rightarrow S^{\prime} \mid E_{i}\right)>P\left(S \rightarrow S^{\prime}\right)
$$

The above formulation suggests identifying new physicalstimulus technologies that shift digital citizenship state to a smarter level. Learners are part of a cyber-physical continuum for education where a spectrum of blended augmented experiences offer new opportunities for instructional practices. In this context, connected devices consist of internetconnected sensors attached to physical phenomena and linked to web applications that collect and curate data. The success of this educational convergence depends on engaging learners along this cyber-physical continuum, through contemporary instructional approaches such as experiential learning, gamification, inquiry-based and problem-based learning to cultivate Digital Smart citizenship competences. Physical phenomena are visualized via appropriate hardware, interfaces and applications that develop tangible learning to elevate digital smart citizenship competence levels. This is achieved through a visual modeling language based on concept maps [16], that conveys a qualitative vocabulary as the basis for learners to abstract quantitative data about physical phenomena into conceptual knowledge [2]. Cyberphysical learning employs this visual structure that link physical entities to present an interactive learning platform to learn from and about physical processes in view of adopting desired behaviors and developing new digital smart citizenship competences.

The following section outlines the proposed model and complementing system architecture for realizing the potential of cyberphysical learning.

\section{MODEL AND SYSTEM ARCHITECTURE}

In Smart City contexts, there is a proliferation of embedded IoT devices with sensing capabilities that capture large amounts of data across the different dimensions of Smart Cities, which can reveal citizens' behavior. For example, existing devices such as power sensors have built-in features to capture power consumption that could be turned into usage behavioral patterns. Sensors perform automated measurements of environmental variables and control some desired aspects of the environment through autonomous or directly controllable probing and actuating activities. In addition, these entities incorporate wireless connectivity modules allowing both collocated devices and citizens (via their smartphones) to seamlessly interact. In this context, these synergies are controlled by a model library, and can be utilized to represent the incremental understanding of cyberphysical learning that needs to be attained for achieving the next level in the digital smart citizenship competences of each 
citizen(-learner), and can also be used as the means to build a portfolio of such competences for reflection. Each instance of this model library is associated to one or more scenarios that simulate the model instance to predict the citizenlearner behavior. In such scenarios, citizens-learners achieve the desired level of competence when the simulator generates the intended behaviour. Autonomous processing of data, response and control allow actors of this cyberphysical ecosystem to input change into selected models. Each actor is outfitted with a unique identifier through Internet of Things technologies to get the ability to transmit data within the cyberphysical network and beyond [22]. Examples of this cyberphysical configuration where the above qualitative modeling approach can be applied extend to smart communications, smart energy and resource management, as well as smart transportation. All of which require citizens' involvement through a progressive learning process.

\subsection{Smart Object Virtualization}

Integrating new generation of IP-enabled objects are propelling IoT applications across a wide spectrum of domains. Embedded 6LoWPAN Wireless sensors with IPv6 perceive environmental parameters and bring objects to Internet. A smart object is defined with four embedded or augmented capabilities, namely: unique identification, processing, storage and communication. Figure 2 shows this virtualization composed of sensor/actuator module, CPU module, battery module, and communication module (radio). CPU provides limited storage and processing capabilities. This module runs the device interface and control components as well as lightweight RESTful web services. The device interface includes an analog to digital transformers with data encoder facilities that provide online/offline data access functionalities. Data is stored in lightweight SQLite database system. Web service layer defines data access operators, control activities, event-trigger mechanisms, and QoS parameters. A physical sensor/actuator node is abstracted as a RESTful web service. Physical devices are thus packaged into services access via short URLs. These devices may be connected directly or indirectly to IoT platform via a gateway bridge. In the latter case, sensors and actuators communicate using wireless protocols such as Zigbee or bluetooth but use the gateway to perform data transfers in HTTP format via built-in light-weight web services within the gateway. In any case though, communication between physical nodes is abstracted by RESTful web services.

\subsection{Cyberphysical Community}

Figure 3 and Figure 4 show a software representation of a community platform where data is exchanged across RESTful web services in JSON format. A cyberphysical community is built to divide citizen-members into model-driven aggregation of heterogeneous resources. Interactions are triggered by model-defined rules. Learning models within the library define actor interactions in the community as illustrated in Table 2 . The model library is provided by resource manufacturers, service-providers or other third-party stakeholders (e.g., education providers) seeking to increase digital smart citizenship competences of their target group base. Our approach is to enable an integrated system whose main objective is to stimulate citizens to learn about adopting behavioral activities in order to raise digital smart citizenship competences, by progressively reflecting on the knowledge

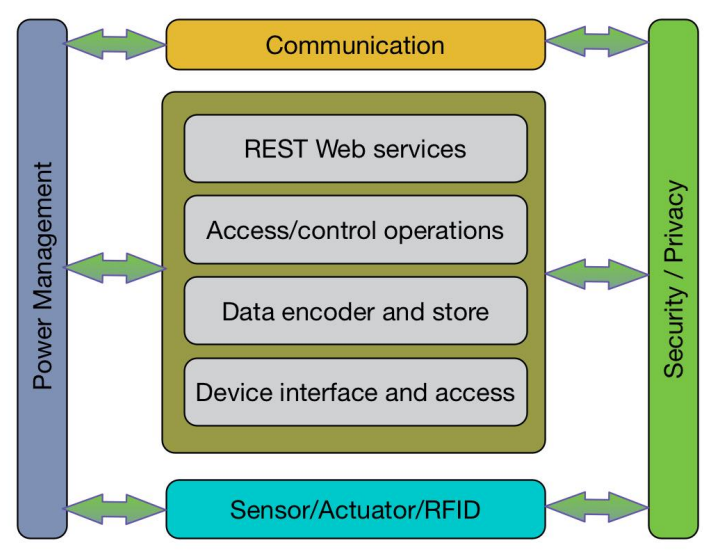

Figure 2. Smart object virtualization

acquired from the deployed models involving surrounding physical resources. Sensing devices monitor physical environments and involve citizens in social network-like synergies to guide them into activities driven by scenarios that are associated with each learning model.

\subsection{Learner Profile}

Each citizen-member of the cyberphysical network is described by its updatable and extendable profile. Usage of the profiles makes it possible to "individualize" proactive learning. Model configurations is driven by the analysis of members' profiles, such as the parameters of smart objects and behaviors of members. Table 1 and Table 2 provide respectively an overview of smart object and learner nodes within a cyberphysical network. Events occurring from network members trigger gap analysis to optimize behaviorallearning criteria. Progressive learning is advocated to incite behavioral change based on information related to antecedents and consequences stored in each network member profile.

Table 1. Smart-object member profile

\begin{tabular}{|l|l|}
\hline Category & Description \\
\hline \hline Context & Perceived phenomenon information \\
\hline Measurement & Data source pattern \\
\hline Consequence & Parameter re-configuration \\
\hline
\end{tabular}

Table 2. Cyberphysical learner profile

\begin{tabular}{|l|l|}
\hline Category & Description \\
\hline \hline Current behavior & Current competence \\
\hline Target behaviour & Target competence \\
\hline Learning path & Cyberphysical learning environments \\
\hline
\end{tabular}

\subsection{Progressive Learning}

Behaviorists such as B. F. Skinner state that complex behavior is learned gradually through the modification of simpler behaviors [17]. We define a learning path $\mathcal{L P}$ as a route, 


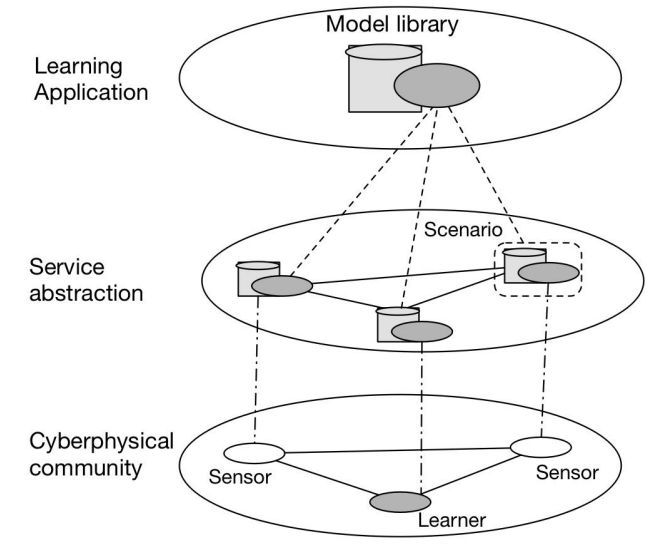

Figure 3. Cyberphysical social interactions

across an ordered set of cyberphysical learning environments $\operatorname{Env}_{i}$.

$$
\mathcal{L P}=\ldots\left\{E n v_{1}, E n v_{2}, \ldots E n v_{p}\right\}
$$

Each cyberphysical learning environment $E n v_{i}$ is composed of a cyberphysical community represented by the set of data sources $\mathcal{D} \mathcal{S}_{i}$ generated by the actors of the community, within a model-based learning structure, as well as a set of scenarios $S_{i}$ and a targeted competency $C_{i}$.

$$
\operatorname{Env}_{i}=\bigsqcup_{1 \leq j \leq p} \mathcal{D} \mathcal{S}_{j}
$$

Digital smart citizenship competence building proceeds progressively across cyberphysical environments with increasing data set sources. Cyberphysical learning models contributing to digital smart citizenship involve interconnected smart objects within a progressively-scaled cyberphysical community where resulting dynamics are dictated by learning scenarios to assert a prescribed competency. A learning model $M_{i}$ is defined as a function of a given cyberphysical environment $E n v_{i}$ that is mapped to a targeted competence $C_{j}$ through instructional scenarios $S_{i j}$ :

$$
M_{i}:\left(\operatorname{Env}_{i}, C_{j}\right) \rightarrow S_{i j}
$$

A model is bound to a cyberphysical learning environment. In this context, model-based learning prevents the information flow to outpace the capacity of learners in absorbing the next digital smart citizenship competence within a given cyberphysical learning environment. Hence, learning models start with basic data sources and proceed to more complex ones along a curriculum-like structure, termed as "progressive learning path". Algorithm 1 shows a process that leads to inductive learning of concepts from examples materialized by data flows distributed over time.

The following section will present a case study for presenting and preliminarily evaluating the aforementioned model for supporting digital smart citizenship competences.

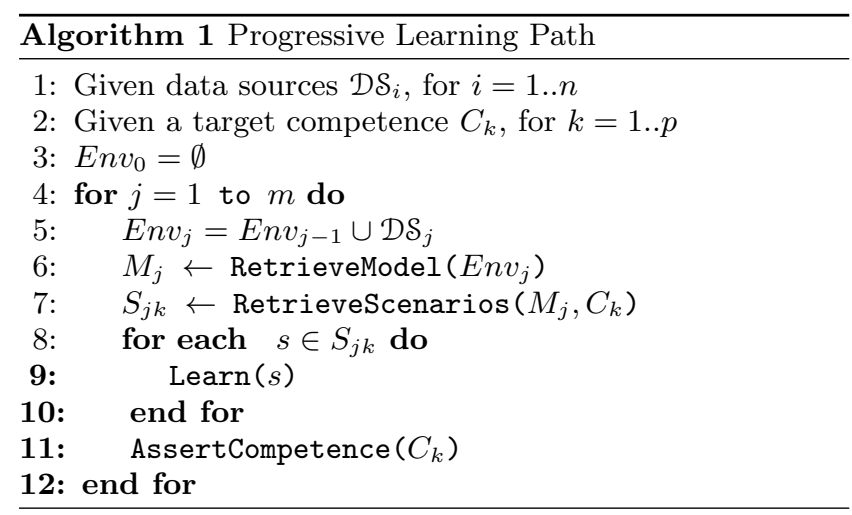

\section{CASE STUDY AND ANALYSIS}

This case study focuses on energy consumers, who form an integral part of the upcoming smart grid within smart cities, as they engage in utilizing new information and adopting new behavior to better balance energy supply and demand. The success of this evolution relies on the transformation of passive end-users into actively engaged digital smart citizens. We propose the adoption of our proposed cyberphysical learning approach to drive this transformation by integrating Internet of Things technologies and resulting data analytics into household practices. This integration enables energy suppliers understand and affect their customers' behaviour to embrace an active role into smart grid operations. In an attempt to assist utilities understand how a customer may act in the future smart grid, this case study investigates the deployment of data intensive customer segmentation across a set of increasing levels of digital smart competences. Utilities gain customer intimacy through incentive rewards such as energy tariff models for raising their digital smart competence levels.

Discerning how much power is being used across home appliances (called energy disaggregation) is achieved through non-intrusive IoT represented by smart plugs as illustrated earlier in Figure 1. Data is streamed over a cloud-platform via load-monitoring services similar to the one depicted in Figure 3. Thus, energy disaggregation identifies and tracks major appliances at home -including air-conditioning, electric heating, pool pumps, refrigerators, electric water heaters, electric car charging, etc - to push personalized learning scenarios that lead to saving tips or reward incentives, via a client mobile app or a Web-based portal. A simulation tool evaluates the operations of a home under various hypothetical "what if" scenarios to predict insights that drive utility customers to develop smart grid competences.

In Sweden, energy providers offer retail consumers electricity contracts based on real-time pricing following DemandResponse schemes which is a form of supply-demand price adjustments in energy markets [4]. As electrical energy cannot be stored in large quantities with current technology, the balance of the electricity supply has to be maintained continually by adjustments in supply to meet an unrestricted customer demand. Hence, real-time pricing appears a fair and flexible scheme over a rigid time-of-day use scheme which projects peak-demand trends. However, real-time tariffs have to be accompanied by appropriate home automation technologies on the one hand and the necessary digital smart competences in order for consumers to understand and mod- 


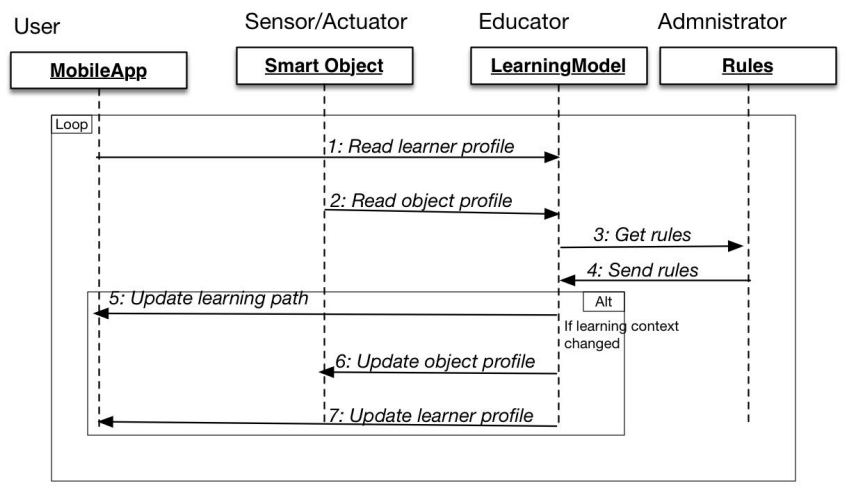

Figure 4. Cyberphysical learning workflows

ify their daily consumption behaviour. We have conducted an experiment where smart-plugs augment home appliances with IoT capabilities, allowing the customer to be informed continually of the cost of a kiloWatt hour (kWh) at the time of use. The home is also fitted with a smart-meter which data is monitored via a led-light reader to capture overall real-time power usage. Next, we illustrate the competencedriven cyberphysical learning model discussed throughout this paper in the context of a smart-home energy management. This envisioned case study illustrates the profound transition for energy management patterns from the conventional centralized infrastructure towards the autonomous demand-response and cyber-physical energy systems. The advocated learning scenarios discussed next and further illustrated in Figure 5 contribute to the progressive learning build-up of digital smart-citizenship.

\subsection{Competence 1: Energy use data}

As described earlier, a learning path is composed of an ordered set of cyberphysical learning environments. The initial instance of such environment in this case study consists in monitoring data from a washing-machine. The targeted outcome from this situated learning process is an increased awareness on the value of energy data and an ability to assess energy-consumption patterns to gain competence into embracing energy-savings programs.

Model 1: Washing-machine

This model includes the washing machine as a cyberphysical learning environment and strives to build Competence 1 through the following scenarios.

Scenario 1: Visualization of current power data: Displays the current power consumption and costs using data from washing-machine and energy-retailer. A simulation model calculates energy consumption and predicts energy savings, resulting from comparative or model household. The purpose of this task is to provide statistically relevant data from typical communities with respect to washing-machine power usage. This scenario introduces household inhabitants to the power of visual analytics.

Scenario 2: "What-if" appliance is started at an alternative time: Displays measures and schedules to match against user-specified schedules via a schedule wizard, which provides a graphical view where user inputs defining custom schedules are streamlined (and contrasted with schedules defining DR behavior of appliances). The scenario would suggest to move laundry task to off-peak hours or along DR programs to meet some rewarding incentives. This behaviouralshift would raise the competence level in smart digital citizenship, if adopted. This scenario develops active digital footprints whereby user data is released deliberately through an enhanced intimacy between users and physical assets.

Scenario 3: "What-if" appliance uses alternative programs: Provides predictive analytics with various wash/rinse temperature settings to heat the water for washing clothes, to engage user in energy-saving alternatives by just changing the temperature setting. The scenario displays $\mathrm{kWh}$ of electricity used for each alternative along with cost per load and cost per year. Current load is contrasted to position the current behaviour of user profile. A target behaviour is proposed to incite user to experience a new energy-saving setting for the washing activity. This scenario drives users into adopting an intervening role in digital smart citizenship.

\subsection{Competence 2: Energy use footprint}

The consumer is presented with information about his global home energy consumption. The smart-meter data source is added to the cyberphysical learning environment, which includes instant power data. This data could be dispatched periodically or upon request. The smart meter provides its data to the cloud-based model-library through a meter service module where the meter records its measurements in the form of index (one per tariff period type). It also exposes a service interface for delivering information (index, tariff period, etc.) to devices in the cyberphysical community (currently limited to washing-machine and consumer members). This information may be directly received, treated, stored and displayed on a dedicated display or user's mobile app, either in $\mathrm{kWh}$ or in $€$. It may also be collected by a cloud-hosted Web application aggregating data from community members to contrast them against individual energy-footprint contribution. 


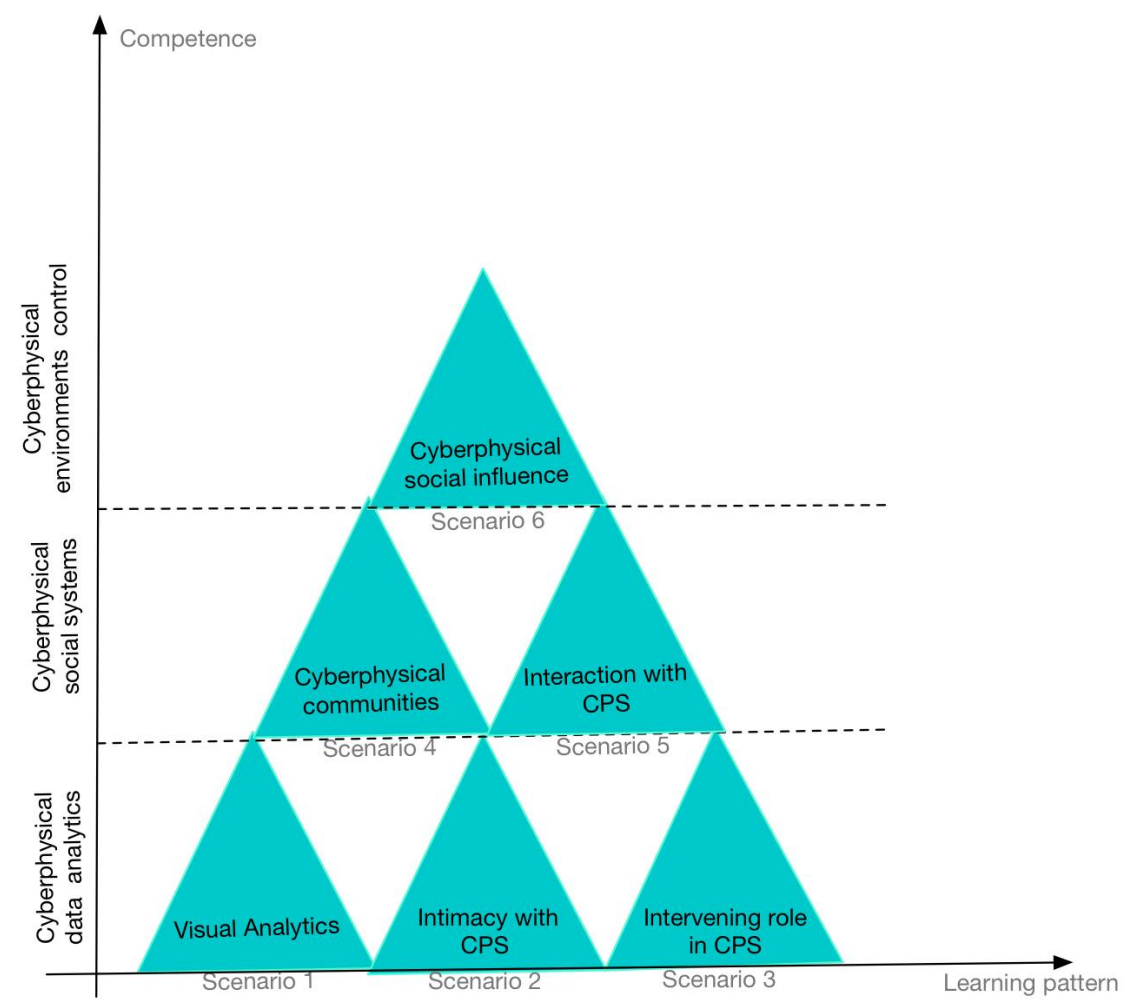

Figure 5. Progressive learning-path build-up in the proposed case-study

\section{Model 2: Smart-meter and washing-machine}

This model augments the cyberphysical learning environment with a smart meter in addition to the washing machine and strives to build Competence 2 through the following scenarios.

Scenario 4: Display global and per appliance energy consumption and costs, either realized and/or forecasted: The aim of this scenario is to provide consumer with information on the current situation of her/his home consumption and in particular one of the smart appliances. Consumer is presented with information about the global current home energy consumption and the appliance contribution. Information is provided by a dedicated service, which retrieves data from the smart-meter and, integrates data measured by the washing-machine's smart plug. Energy rates service provided by retailer is composed with data retrieval service to display costs under different tariff periods in order to educate consumers on a more efficient and rationale use of energy. The targeted behavioural trend with this scenario is the reduction of global energy by leveraging smart appliance footprints. Consumers are educated to select the parameters of the appliances (in this case washing-machine cycles) by providing feedback on forecasted costs and energy consumption. This scenario introduces users into cyberphysical social communities, which are poised to shape future smart cities.

Scenario 5: Devices monitoring: This scenario expands consumer's abilities to manage connected devices in his cyberphysical community to monitor their status and functionalities. The user can receive remotely on his mobile or a
Web app the status of the devices; in this case his washingmachine to let him perform monitoring operations. This scenario works on devices which operations are remotely accessible, such as the current phase of the device (e.g. for a washing machine Heating, Rinse, Spin, etc), the selected program, the selected options (temperature, duration, spin, etc), special functions (pre-wash, extra-rinse, etc), and the remaining time to end the current cycle. On-off options can always be provided via smart-plugs on devices which do not provide elaborated remote monitoring services. Similarly to the way Internet has changed our interaction with each other, this scenario develops new perceptions to change the way citizens interact with the physical world.

\subsection{Competence 3: Energy use control}

This competence empowers self-discipline in maintaining a threshold of energy use across a range of appliances.

Model 3: Smart-meter, washing-machine and dryer

This model considers a cyberphysical learning environment with a combination of three physical elements: washing machine, dryer and smart-meter, and strives to build Competence 3 through the following scenarios.

Scenario 6: "What-if" total power exceeds a preset threshold: In this scenario, user chooses preset thresholds of global energy use. Each smart appliance applies statistical analysis on past data to estimate the maximum power that would be consumed during its next cycle, and determines whether there exists a risk or threat to exceed the user threshold. This threshold trains consumers on the potential gains from 
Interaction with smart devices raises individual digital competence

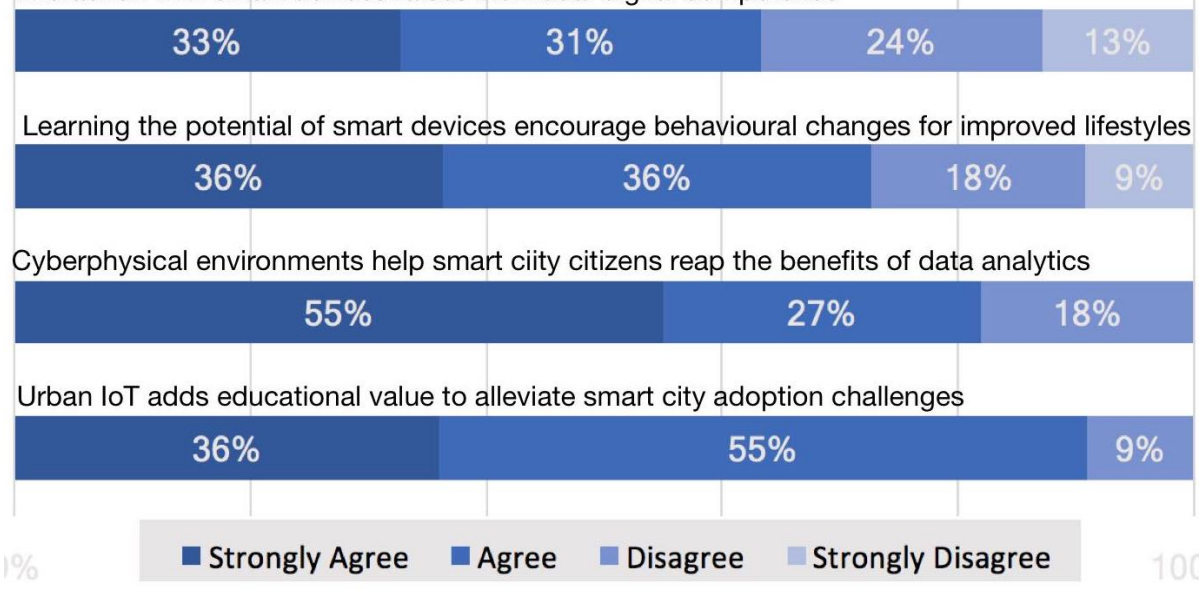

Figure 6. Survey results on cyberphysical learning environments support for digital smart citizenship

contracted subscribed power. A warning is emitted and displayed on the appliance or other consumer interface(s) such as mobile app. The appliance works in tandem with other appliances to cooperate on collaborative settings that are deemed to bring energy use below the preset threshold. In this case, dryer and washing machine negotiate their programs to calibrate jointly their energy use. For example, heating temperature is set in both devices accordingly to optimize washing/drying operations while complying with power-use threshold. Consumer is presented with options to combine both programs and invited to select adequate options. This level of energy-aware engagement with appliance settings could contribute to driving users to the next era of digital smart citizenship. This scenario allows citizens to influence cyberphysical communities adopting a desired pattern that drives values for users.

An experiment that identifies users' believes to employ cyberphysical environments for learning for enhancing their level of digital smart citizenship was conducted. The above case study and related scenarios were depicted as an illustration to respondents of the survey instrument that tests the hypothesis whereby cyberphysical environments have a positive effect on users' learning towards digital smart citizenship. A four-point Likert scale, with 1 being the negative end of the scale (strongly disagree) and 4 being the positive end of the scale (strongly agree), was used to examine participants' responses to the items of the survey. The data for this study was anonymously collected from Computer Science students who are aware about contemporary smart-city developments, and living in a residential area of a medium size city in Sweden. 65 completed questionnaires were collected, among which 55 of them were valid questionnaires. The results displayed in Figure 6 show that the hypothesis was significantly supported, with a statistically positive effect on users' attitude towards embracing cyberphysical learning environments to foster digital smart competences. Particularly, the obtained results from the two last questions of this preliminary survey show the benefits of data analytics to empower future digital smart citizens on one hand; and on the other hand, reveal an awareness on the prominent role of IoT to alleviate challenges towards smart-city adoption.

\section{RELATED WORK}

There has been recent investigations into raising citizens' aptitude to meet the digital profile of smart citizens, through the development of computational-education environments. A future education has been envisioned in [21] which emphasizes the idea of 'smart citizens'. IBM's Smarter Education [10] program is based on a similar motivation but highlights the availability of real-time data enabled by new technologies for driving the educational continuum. This trend aims at extending IBM's global smart cities programs into reimagining and reconfiguring education as data-based platforms of real-time monitoring and measurement, where learners are increasingly treated as 'data objects' along with environmental assets, which actions can be altered through programming the learning environment. Along the same line, Microsoft has been examining how governmental and civil society organizations are working within local smart cities initiatives to develop citizen's capacities as active 'smart citizens' [11]. Competences include technical data skills facilitated by computational urbanism such as 'smart homes' and participation in 'civic coding' on behalf of the city. Microsoft CityNext initiative on education connects 'cloud, Big Data, mobile and social' technology trends to the smart city and to education [11]. To thrive, "smart cities need to provide access to powerful learning devices, tools, and apps that empower education" argues Microsoft's white paper. Other commercial computing firms have launched projects promoting their products for smart cities, including Cisco, Intel, and Siemens, many are linked to huge urban projects building within new smart cities, such as Songdo in South Korea, PlanIT Valley in Portugal and Masdar in Abu Dhabi, but much more commonly in the 'upgrading' of existing urban infrastructure [20], such as Stockholm, Helsinki, Amsterdam, Seattle and Singapore. To achieve these urbanization developments, an efficient cooperation between inhabitants, industry and technology players is expected, prompting shifts in learning processes which evolved outside traditional classrooms towards data-intensive approaches. These processes are turning tangible interfaces into learning tools through real-time feedback from physical instructional assets that can 'teach' how they are to be used and deliver personalized educational experience [13]. 


\section{CONCLUSION}

Smart cities raise the expectations for citizen's engagement and increase their digital footprint. As manufacturers brainstorm, envision, and plan smart city developments, citizens found themselves pruned out and set aside for later or forgotten altogether. Yet, the success of smart cities rely on general citizens' engagement into these spaces enacted by an assemblage of programmed technologies and data performed from technical experts. We revealed a computational representation of these spaces using advances in Internet of Things (IoT) that shape cyberphysical learning environments. We also advocated progressive learning processes within these environments, following a model-based learning scheme that integrates physical assets as sources of instruction. The proposed algorithm asserts digital competencies within specific smart city contexts. A case study illustrating the proposed cyberphysical learning approach is discussed and analyzed along contemporary smart-grid developments, where smart-homes augmented or fitted with IoT technologies empower inhabitants to feedback on their energy usage and adopt behavioral changes that expand their digital smart citizenship competences. Ongoing work aims at demonstrating the proposed cyberphysical learning approach within a prototype implementation of a connected home to validate incremental advancements in smart digital citizenship competence.

\section{ACKNOWLEDGMENTS}

The first author's contribution in this work has been partially funded by Västra Götaland Region, as a part of the research project on smart grid Kraftsamling Smarta Nät 2015-2016 (dnr MN 39-2015), and partially supported by SP Sveriges Tekniska Forskningsinstitut AB as well as ELIQ AB (energy management company). The second author's contribution in this work has been partially funded by the Greek General Secretariat for Research and Technology, under the Matching Funds 2014-2016 for the EU project "Inspiring Science: Large Scale Experimentation Scenarios to Mainstream eLearning in Science, Mathematics and Technology in Primary and Secondary Schools" (Project Number: 325123).

\section{REFERENCES}

[1] L. Anthopoulos. Understanding the smart city domain: A literature review. In Transforming City Governments for Successful Smart Cities, pages 9-21. Jan. 2015.

[2] B. Bredeweg and K. D. Forbus. Qualitative Modeling in Education. AI Magazine, 24(4):35, Dec. 2003.

[3] J. S. Brown, A. Collins, and P. Duguid. Situated Cognition and the Culture of Learning. Educational Researcher, 18(1):32, 1989.

[4] J. Campillo, F. Wallin, and I. Vassileva. Economic impact of dynamic electricity pricing mechanisms adoption for households in Sweden. In Proceeding of the World Renewable Energy Congress, 2013.

[5] G. Clemitshaw. Children, citizenship and environment: nurturing a democratic imagination in a changing world. by bronwyn hayward. British Journal of Educational Studies, 62(1):85-86, 2014.

[6] A. Cocchia. Smart and digital city: a systematic literature review. Smart city. Springer International Publishing, pages 13-43, 2014.
[7] E. Commission. Integrated sustainable urban development, cohesion policy 2014-2020. European Commission: Brussels, Belgium, 2012.

[8] R. P. Dameri. Searching for Smart City definition: a comprehensive proposal. International Journal of Computers \& Technology, 2013.

[9] S. Das. Personalise through the internet of things. Kantar TNS. Retrieved from: http://www.tnsglobal.co.uk/thoughtleadership/personalise-through-internet-of-things, 2016., 2016.

[10] I. S. Education. Education for a Smarter Planet: The Future of Learning. Available at: http://www.redbooks.ibm.com/redpapers/pdfs/redp4564.pdf (accessed 5 January 2016).

[11] M. Education. Educated Cities. Available at https://enterprise.microsoft.com/enus/industries/citynext/educated-cities/ (accessed Janaury 5, 2016).

[12] European Commission. The european digital competence framework for citizens. Publications Office of the European Union, 2016.

[13] F. Gianni, S. Mora, and M. Divitini. IoT for Smart City Learning - Towards Requirements for an Authoring Tool. Proceedings of the First International Workshop on Smart Ecosystems cReation by Visual dEsign co-located with the International Working Conference on Advanced Visual Interfaces, 2016.

[14] J. R. Gil-García, T. A. Pardo, and T. Nam. What makes a city smart? Identifying core components and proposing an integrative and comprehensive conceptualization. Information Policy, 20(1):61-87, 2015.

[15] C. Kyriazopoulou. Smart City Technologies and Architectures - A Literature Review. SMARTGREENS, 2015.

[16] J. D. Novak and D. B. Gowin. Learning how to learn. Cambridge University Press, 1984.

[17] B. F. Skinner. Teaching machines. Science, 1958.

[18] A. N. special issue. Circular economy: Getting the circulation going. Nature, 531(7595):443-446, Mar. 2016.

[19] U. N. (UN). Press release of the world urbanization prospects 2014. United Nations - Department of Economic and Social Affairs. Retrieved from http://tinyurl.com/j3azkyw., 2014.

[20] A. Wiig, T. Shelton, and M. Zook. "The 'actually existing smart city" by Alan Wiig. Cambridge Journal of Region. Available at http://cjres.oxfordjournals.org/content/8/1/13 (accessed Janaury 5, 2017), 2015.

[21] B. Williamson. Educating the smart city: Schooling smart citizens through computational urbanism. Big Data \& Society, 2(2), 2015.

[22] L. Yang, W. Li, Y. Ge, X. Fu, R. Gravina, and G. Fortino. People-Centric Service for mHealth of Wheelchair Users in Smart Cities. Internet of Things Based on Smart Objects, Technology, Middleware and Applications, 2014. 